\title{
Analysis and Simulation on an ISFET with Back- Gated Structure and High-Mobility Channel Material
}

\author{
X. Wu, A. Z. Jia \\ School of Microelectronics and Solid-State Electronics \\ University of Electronic Science and Technology of China \\ China
}

\begin{abstract}
A back-gated structure for ion-sensitive field-effect transistor (ISFET) has been proposed. The characteristics of the device based on this proposed structure and with high-mobility channel material have been simulated and analyzed by Silvaco TCAD. The modeling and simulation methodologies have been investigated, and an average sensitivity approximate $49.56 \mathrm{mV} / \mathrm{pH}$ is obtained. The detectable average sensitivity of drain current is about $54.23 \mu \mathrm{A} / \mathrm{pH}$ with a high saturated drain current which is at the magnitude of $10^{-4} \mathrm{~A}$, indicating a promising application in biological, biochemical and medical fields.
\end{abstract}

Keywords-ISFET; modeling and simulation; high mobility; back-gated; average sensitivity

\section{INTRODUCTION}

The ion-sensitive field-effect transistor (ISFET) has received a lot of interest, since it was first reported by Bergveld in 1972[1]. Due to the promising application in biological, biochemical and medical detection [2-4], the ISFET based on a metal-oxide-semiconductor field-effect transistor (MOSFET) structure has been extensively studied. In particular, much effort has been made on investigating $\mathrm{pH}$ sensitive ISFETs, which device structures and $\mathrm{pH}$-sensing membranes have been studied to improve the sensitivity and stability of the ISFETs [4-6]. It is well known that the gate dielectric is in direct contact with the electrolyte solution, which determines the beginning sensitivity of these devices. As $\mathrm{SiO}_{2}$ gate dielectric shows low response sensitivity and poor stability, other inorganic materials, such as $\mathrm{Al}_{2} \mathrm{O}_{3}$ [5], $\mathrm{Si}_{3} \mathrm{~N}_{4}$ [6,7] and $\mathrm{Ta}_{2} \mathrm{O}_{5}$ [8] with enhanced stability and sensitivity have been investigated. Moreover, the drain-source voltage of the ISFET based on a MOSFET structure also affects the ionic charges with a horizontal electric field in the electrolyte solution, resulting in a non-uniform charge distribution at the electrolyte-insulator interface. The operation mechanism of a pH-sensitive ISFET is the change of potential between the electrolyte solution and the gate dielectric surface, thus leading to an increased or decreased output current of the ISFET. Although the impurity doping could improve the properties of semiconductor materials [9-10], the $\mathrm{pH}$-sensitive ISFETs with high-mobility channel materials, such as InSb and graphene [11,12] may have intrinsic advantages of low response delay and high sensitivity, and the output current of the ISFET can be also increased.

We proposed a back-gated ISFET structure, in which the back-gate of thin-film transistor can be fabricated by etching the substrate and the electrolyte solution is separated from the drain/source electrodes. In this paper, the characteristics of the device based on this proposed structure with high-mobility channel material have been investigated by Silvaco TCAD. The simulation is based on the detection of $\mathrm{pH}$, as the $\mathrm{pH}$ sensitivity ISFET has the most accurately model and their physical and chemical behaviour had been studied extensively. The response of $\mathrm{pH}$-sensitive devices in electrolyte solutions is commonly explained by using the site-binding theory [13].

\section{DEVICE STRUCTURE AND MODEL}

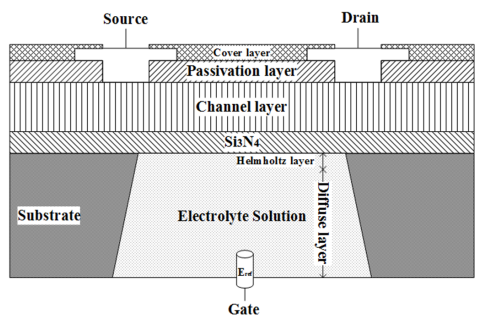

FIGURE I. SCHEMATIC LAYOUT OF THE BACK-GATED ISFET STRUCTURE.

Figure.1 shows the schematic layout of proposed backgated ISFET structure. The high-mobility n-channel layer is deposited on a $\mathrm{Si}_{3} \mathrm{~N}_{4}$ layer with flexible organic substrate, and the gate of ISFET is achieved on the back side by etching the substrate after fabricating drain and source regions on the right side. In this device simulation, InSb with a doping concentration of $10^{17} \mathrm{~cm}^{-3}$ is utilized as channel material. The doping concentration of drain and source regions are $10^{20} \mathrm{~cm}^{-3}$, and the channel length is $6 \mu \mathrm{m}$. The thickness of the channel layer and the gate dielectric after etching is $160 \mathrm{~nm}$ and $40 \mathrm{~nm}$, respectively.

According to the site-binding and electrical double layer theory [7,14], we considered the Helmholtz layer and diffuse layer in the electrolyte solution as two in series capacitors, thus the potential drop $\psi$ between the electrolyte solution and the surface of the $\mathrm{Si}_{3} \mathrm{~N}_{4}$ dielectric can be expressed as:

$$
\psi=\frac{\sigma C_{H} C_{D}}{C_{H}+C_{D}},
$$

where $C_{H}$ is the Helmholtz layer unit-area capacitance, $C_{D}$ is the diffuse layer unit-area capacitance and $\sigma$ is the charge density at the electrolyte-insulator interface, respectively. The Helmholtz layer unit-area capacitance $\mathrm{C}_{\mathrm{H}}$ can be derived by considering the in series of the inner and outer Helmholtz layers: 


$$
C_{H}=\frac{\varepsilon_{I H P} \varepsilon_{O H P} \varepsilon_{0}}{\varepsilon_{O H P} d_{I H P}+\varepsilon_{I H P} d_{O H P}},
$$

where $\varepsilon_{\mathrm{IHP}}$ and $\varepsilon_{\mathrm{OHP}}$ are the inner and outer Helmholtz plane dielectric constants, $\varepsilon_{0}$ is the permittivity of vacuum, $\mathrm{d}_{\mathrm{IHP}}$ and $\mathrm{d}_{\mathrm{OHP}}$ are the insulator-non-hydrated ion and the insulatorhydrated ion distances, respectively. The diffuse layer unitarea capacitance $C_{D}$ is given by [14]:

$$
C_{D}=q \sqrt{\frac{2 \varepsilon_{e} c_{b}}{k T}},
$$

where $\mathrm{T}$ is the temperature, $\mathrm{k}$ is the Boltzmann constant, $\mathrm{q}$ is the electronic charge, $\varepsilon_{\mathrm{e}}$ is the permittivity of the electrolyte solution; $\mathrm{c}_{\mathrm{b}}$ is ion concentration in the electrolyte solution. The charge density $\sigma$ at the electrolyte-insulator interface is given by [14-16]:

$$
\begin{gathered}
\sigma=q N_{s}\left(\frac{\left[H^{+}\right]_{b}^{2} \exp \left(-\frac{2 q \psi}{k T}\right)-K_{A-} K_{A+}}{\left[H^{+}\right]_{b}^{2} \exp \left(-\frac{2 q \psi}{k T}\right)+K_{A+}\left[H^{+}\right]_{b} \exp \left(-\frac{q \psi}{k T}\right)+K_{A-} K_{A+}}\right) \\
+q N_{n}\left(\frac{\left[H^{+}\right]_{b}^{2} \exp \left(-\frac{q \psi}{k T}\right)}{\left[H^{+}\right]_{b}^{2} \exp \left(-\frac{q \psi}{k T}\right)+K_{B}}\right)
\end{gathered}
$$

where $\left[\mathrm{H}^{+}\right]_{\mathrm{b}}=10^{\wedge}(-\mathrm{pH})$ is the concentration of $\mathrm{H}^{+}$at the bulk electrolyte solution, $\mathrm{N}_{\mathrm{s}}$ and $\mathrm{N}_{\mathrm{n}}$ are the surface densities of the silanol sites and the primary amine sites, $\mathrm{K}_{\mathrm{A}-}, \mathrm{K}_{\mathrm{A}+}, \mathrm{K}_{\mathrm{B}}$ are the dissociation constants of the silanol sites and the primary amine sites, respectively. Therefore, the relation between $\psi$ and $\mathrm{pH}$ can be obtained by solving the equations of eqn(1)-(4).

The general threshold voltage $\mathrm{V}_{\mathrm{T}}$ of a MOS structure is given by:

$$
V_{T}=V_{F B}+V_{o X}+2 \phi_{F}
$$

where $\mathrm{V}_{\mathrm{FB}}$ is the flatband voltage, $\mathrm{V}_{\mathrm{OX}}$ is the voltage drop at the gate dielectric, $\Phi_{\mathrm{F}}$ is the Fermi-potential. The threshold voltage $\mathrm{V}_{\mathrm{T}}{ }^{*}$ of this proposed device can be expressed as [16]:

$$
V_{T}^{*}=E_{r e f}+\chi_{s o l}-\psi+V_{T},
$$

where $E_{\text {ref }}$ is the potential of the reference electrode, $\chi_{\text {sol }}$ is the surface dipole potential of the gate insulator-electrolyte, which are both constants. The parameters [14-16] used in simulation are list in table.1.

TABLE I .THE PARAMETERS USED IN THIS DEVICE SIMULATION.

\begin{tabular}{|c|c|c|c|}
\hline $\boldsymbol{\varepsilon}_{\mathbf{I H P}}$ & 32 & $\mathrm{~K}_{\mathrm{A}+}$ & $63.1 \times 10^{-9}$ \\
\hline $\boldsymbol{\varepsilon}_{\mathbf{O H P}}$ & 32 & $\mathrm{~K}_{\mathrm{B}}$ & $1 \times 10^{-10}$ \\
\hline $\mathbf{d}_{\mathbf{I H P}}(\mathbf{n m})$ & 0.1 & $\mathrm{~N}_{\mathrm{s}}$ & $3 \times 10^{-18}$ \\
\hline $\mathbf{d}_{\mathbf{O H P}}(\mathbf{n m})$ & 0.3 & $\mathrm{~N}_{\mathrm{n}}$ & $2 \times 10^{-18}$ \\
\hline $\boldsymbol{\varepsilon}_{\mathbf{e}}$ & 78.5 & $\mathrm{E}_{\mathrm{ref}}$ & 0.205 \\
\hline $\mathbf{c}_{\mathbf{b}}(\mathbf{1} / \mathbf{m o l e s})$ & 0.1 & $\chi_{\mathrm{sol}}$ & $3 \times 10^{-3}$ \\
\hline $\mathbf{K}_{\mathbf{A}-}$ & 15.8 & $\mathrm{pH}$ & $3,5,7,9,11$ \\
\hline
\end{tabular}

\section{RESULTS AND DISCUSSION}

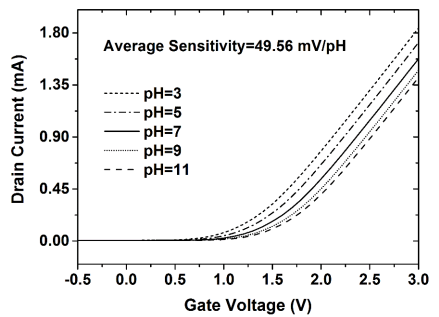

FIGURE II. IDS-VGS TRANSFER CHARACTERISTICS WITH DIFFERENT PH MEASURED AT VDS=0.5V OF THE BACK-GATED ISFET.

The $\mathrm{I}_{\mathrm{DS}}-\mathrm{V}_{\mathrm{GS}}$ transfer characteristics curves with different $\mathrm{pH}$ measured at $\mathrm{V}_{\mathrm{DS}}=0.5 \mathrm{~V}$ of proposed back-gated ISFET are shown in Figure.2. It can be seen that the threshold voltages of this device increased with the increased $\mathrm{pH}$. The simulation average sensitivity of this device is about $49.56 \mathrm{mV} / \mathrm{pH}$, which is in the range of 25 to $58 \mathrm{mV} / \mathrm{pH}$ with a potential/pH response of silicon nitride devices [7]. It is also of interest to note that the use of InSb material can lead a low threshold voltage compared with silicon ISFET at the same doping concentration of channel. As the Fermi-potential $\Phi_{\mathrm{F}}$ of a Ptype semiconductor can be expressed as:

$$
\phi_{F}=\frac{k T}{q} \ln \frac{N_{A}}{n_{i}},
$$

where $\mathrm{N}_{\mathrm{A}}$ is the doping concentration of channel, $\mathrm{n}_{\mathrm{i}}$ is intrinsic carrier concentration of the semiconductor. The intrinsic carrier concentration of InSb and silicon is $1.92 \times 10^{16} \mathrm{~cm}^{-3}$ and $1.43 \times 10^{10} \mathrm{~cm}^{-3}$, and the work function of InSb and silicon is $4.59 \mathrm{eV}$ and $4.17 \mathrm{eV}$, respectively. Therefore, it can be obtained from eqn(6) and eqn(7) that the InSb ISFET has a low threshold voltage compared with silicon ISFET at the same doping concentration of channel, allowing addition of sensing membranes which can further improving the device sensitivity and enlarging the application fields of the device in biological, biochemical and medical detection.

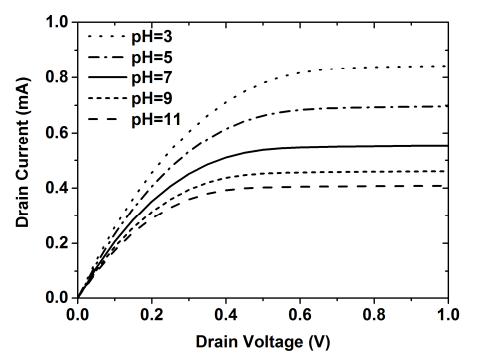

FIGURE III. IDS-VDS CHARACTERISTICS WITH DIFFERENT PH

MEASURED AT VGS $=2.0 \mathrm{~V}$ OF THE BACK-GATED ISFET.

Figure.3. shows the $\mathrm{I}_{D S}-\mathrm{V}_{\mathrm{DS}}$ characteristics with different $\mathrm{pH}$ measured at $\mathrm{V}_{\mathrm{GS}}=2.0 \mathrm{~V}$ of this back-gated ISFET. The drain current of this device decreased with the increased $\mathrm{pH}$, and the detectable average sensitivity of drain current is about $54.23 \mu \mathrm{A} / \mathrm{pH}$. The saturated drain current is at the magnitude of $10^{-4} \mathrm{~A}$. As the saturated drain current is proportional to the mobility, this high-mobility ISFET undoubted has a high drain current, resulting a large detectable current signal of the $\mathrm{pH}$ response. The introduction of high-mobility channel material 
and the etching of substrate as a gate dielectric lead a low response delay and a large detectable current signal, also indicating a promising application in biological, biochemical and medical detection.

\section{CONCLUSION}

In summary, the characteristics of the ISFET device based on proposed back-gated structure and with high-mobility material for the channel have been simulated and analyzed. We focus on the modeling and simulation methodologies of the ISFET device used for the detection of $\mathrm{pH}$, as the accurate model can be achieved by using the site-binding theory. A simulation average sensitivity approximate $49.56 \mathrm{mV} / \mathrm{pH}$ is obtained, and the device exhibits a low threshold voltage compared with silicon ISFET at the same doping concentration of channel. The detectable average sensitivity of drain current is about $54.23 \mu \mathrm{A} / \mathrm{pH}$ with a high saturated drain current which is at the magnitude of $10^{-4} \mathrm{~A}$, exhibiting a large detectable current signal with low response delay. The simulation results of the detection of $\mathrm{pH}$ indicate that this ISFET may have a promising application in biological, biochemical and medical detection by addition of sensing membranes.

\section{ACKNOWLEDGMENTS}

The authors would like to acknowledge the Science and Technology Program of Chengdu for the support. If you have any questions, please directly contact the corresponding author by E-mail at ze.jia@ieee.org.

\section{REFERENCE}

[1] Bergveld, P., Development, operation, and application of the ionsensitive field-effect transistor as a tool for electrophysiology. Biomedical Engineering, IEEE Transactions on, (5), pp. 342-351, 1972.

[2] Bratov, A., Abramova, N. \& Domínguez, C., Investigation of chloride sensitive ISFETs with different membrane compositions suitable for medical applications. Analytica chimica acta, 514(1), pp. 99-106, 2004.

[3] Lee, C.S., Kim, S.K. \& Kim, M., Ion-sensitive field-effect transistor for biological sensing. Sensors, 9(9), pp. 7111-7131, 2009.

[4] Sakurai, T. \& Husimi, Y., Real-time monitoring of DNA polymerase reactions by a micro ISFET $\mathrm{pH}$ sensor. Analytical chemistry, 64(17), pp. 1996-1997, 1992.

[5] Chen, S., Bomer, J.G., Carlen, E.T. \& van den Berg, A., A12O3/silicon nanoISFET with near ideal Nernstian response. Nano letters, 11(6), pp. 2334-2341, 2011.

[6] Niu, M.N., Ding, X.F. \& Tong, Q.Y., Effect of two types of surface sites on the characteristics of Si3N4-gate pH-ISFETs. Sensors and Actuators B: Chemical, 37(1), pp. 13-17, 1996.

[7] Harame, D.L., Bousse, L.J., Shott, J.D. \& Meindl, J.D., Ion-sensing devices with silicon nitride and borosilicate glass insulators. Electron Devices, IEEE Transactions on, 34(8), pp. 1700-1707, 1987.

[8] Kwon, D.H., Cho, B.W., Kim, C.S. \& Sohn, B.K., Effects of heat treatment on Ta2O5 sensing membrane for low drift and high sensitivity pH-ISFET. Sensors and Actuators B: Chemical, 34(1), pp. 441-445, 1996.

[9] Jia, Z., Zhang, M.M. \& Ren, T.L., Modulation Effect of Lead Zirconate Titanate for Zinc Oxide Channel Resistance in Ferroelectric Field Effect Transistor. Ferroelectrics, 421(1), pp. 92-97, 2011.

[10] Jia, Z., Xu, J.L., Wu, X., Zhang, M.M., Zhang, N.W., Liu, J.Z., Liu, Z.W \& Liou, J.J., Metal-Semiconductor-Insulator-Metal Structure FieldEffect Transistors Based on Zinc Oxides and Doped Ferroelectric Thin Films. 2013 MRS Fall Proceedings, 1633, pp. mrsf13-1633, 2014.
[11] Fu, W., Nef, C., Knopfmacher, O., Tarasov, A., Weiss, M., Calame, M. \& Schönenberger, C., Graphene transistors are insensitive to $\mathrm{pH}$ changes in solution. Nano letters, 11(9), pp. 3597-3600, 2011.

[12] Semenov, Y.G., Kim, K.W. \& Zavada, J.M., Spin field effect transistor with a graphene channel. Applied Physics Letters, 91(15), pp. 153105, 2007.

[13] Yates, D.E., Levine, S. \& Healy, T.W., Site-binding model of the electrical double layer at the oxide/water interface. Journal of the Chemical Society, Faraday Transactions 1: Physical Chemistry in Condensed Phases, 70, pp. 1807-1818, 1974.

[14] Martinoia, S. \& Massobrio, G., A behavioral macromodel of the ISFET in SPICE. Sensors and Actuators B: Chemical, 62(3), pp. 182-189, 2000.

[15] Grattarola, M., Massobrio, G. \& Martinoia, S., Modeling H+-sensitive FETs with SPICE. Electron Devices, IEEE Transactions on, 39(4), pp. 813-819, 1992.

[16] Dutta, J.C., Ion sensitive field effect transistor for applications in bioelectronic sensors: A research review, Computational Intelligence and Signal Processing (CISP), 2012 2nd National Conference on. IEEE, pp. 185-191, 2012. 